\title{
5G Multimedia Precision Marketing Based on the Improved Multisensor Node Collaborative Filtering Recommendation Algorithm
}

\author{
Kai Si $\mathbb{D}^{1,2}$ Min Zhou $\mathbb{D}^{1},{ }^{1}$ and Yingfang Qiao ${ }^{2}$ \\ ${ }^{1}$ China University of Mining and Technology, Xuzhou 221116, China \\ ${ }^{2}$ Xuzhou University of Technology, Xuzhou 221116, China \\ Correspondence should be addressed to Min Zhou; xzkdzm@163.com
}

Received 25 October 2021; Accepted 11 November 2021; Published 21 December 2021

Academic Editor: Gengxin Sun

Copyright (C) $2021 \mathrm{Kai} \mathrm{Si}$ et al. This is an open access article distributed under the Creative Commons Attribution License, which permits unrestricted use, distribution, and reproduction in any medium, provided the original work is properly cited.

\begin{abstract}
The rapid development of web technology has brought new problems and challenges to the recommendation system: on the one hand, the traditional collaborative filtering recommendation algorithm has been difficult to meet the personalized recommendation needs of users; on the other hand, the massive data brought by web technology provides more useful information for recommendation algorithms. How to extract features from this information, alleviate sparsity and dynamic timeliness, and effectively improve recommendation quality is a hot issue in the research of recommendation system algorithms. In view of the lack of an effective multisource information fusion mechanism in the existing research, an improved $5 \mathrm{G}$ multimedia precision marketing based on an improved multisensor node collaborative filtering recommendation algorithm is proposed. By expanding the input vector field, the features of users' social relations and comment information are extracted and fused, and the problem of collaborative modelling of these two kinds of important auxiliary information is solved. The objective function is improved, the social regularization term and the internal regularization term in the vector domain are analysed and added from the perspective of practical significance and vector structure, which alleviates the overfitting problem. Experiments on a large number of real datasets show that the proposed method has higher recommendation quality than the classical and mainstream baseline algorithm.
\end{abstract}

\section{Introduction}

With the advent and popularization of the Internet and the rapid development of information technology, the total number of users and business types of operators has also increased [1]. By the end of 2018, the total number of Internet users in China had reached 829 million. In 2018, 56.53 million new Internet users were added, and the Internet penetration rate was $59.6 \%$, an increase of $3.8 \%$ over the end of $2017[2,3]$. With the continuous vigorous development of the communication industry and the gradual maturity of the customer life cycle, relevant Internet enterprises and Internet technologies have sprung up, and operators are facing increasing market competition pressure. However, the marketing mode and marketing business of operators have not changed breakthrough, and the market position and traditional business model are greatly challenged. The external and internal of the enterprise are under great development pressure [4].

The stock maintenance work is not carried out in place, and the understanding of stock users is not deep enough. Furthermore, the online data of stock users are not well used for analysis, resulting in poor matching between sales products and users, no in-depth mining of user needs for marketing, and single marketing mode; recommending sales products to users often does not meet user needs, and the maintenance effect of stock users is general [5]. In addition, there is no unified control over the service quality of stock users. At ordinary times, the maintenance of users is extensive marketing [6]. Policies are issued uniformly, and 
marketing activities are formulated and carried out by multiple teams at the same time [7]. In this way, it could have met the personalized policy needs of different marketing units, but there is no unified communication to record the marketing track of users [8]. Each marketing team did not coordinate and communicate during marketing, and it results in repeated pushing the same marketing activity for users, promoting different marketing promotions to the same user at the same time [9]. Different users under the same user or customers of the same unit received marketing messages with different contents, which made users become confused about $5 \mathrm{G}$ precision marketing, reduce their trust, and become more reluctant to accept and maintain promotion [10].

Common recommendation system algorithms mainly include content-based recommendation algorithm, association rule-based recommendation algorithm, knowledgebased recommendation algorithm, collaborative filtering recommendation algorithm, and hybrid recommendation algorithm [11]. Collaborative filtering recommendation algorithms can be divided into two categories: memorybased collaborative filtering algorithms and model-based collaborative filtering algorithms [12]. Memory-based collaborative filtering uses the nearest neighbor search, which can be divided into user-based collaborative filtering (userbased $\mathrm{CF}$ ) and item-based collaborative filtering (item-based CF) [13]. Memory-based collaborative filtering mainly uses similarity calculation, including Pearson similarity and cosine similarity [14]. The model-based collaborative filtering algorithm establishes a prediction model according to the historical data and then uses the method of machine learning to train the parameters of the model to make the model have the ability to predict. Widely used model-based methods include singular value decomposition (SVD), Bayesian network, implicit factor model (IFM), restricted Boltzmann machine (RBM), factor decomposition machine, Bayesian personalized recommendation, and so on $[15,16]$. The advantages of the memory-based collaborative filtering algorithm are that the algorithm is easy to implement and has certain prediction accuracy and the recommended results have good interpretability $[17,18]$. However, this method also has significant disadvantages: it needs to maintain a similarity matrix, resulting in high computational overhead, difficult to deal with cold start and sparsity problems and poor scalability of the algorithm [19]. The modelbased collaborative filtering method can alleviate the sparsity problem [20]. The model has certain scalability and good predictability, but the disadvantages of this method are as follows: the construction of the model is complex, the time complexity of the algorithm is high, and the recommended results are not reasonably interpretable [21].

The collaborative filtering recommendation algorithm is the mainstream recommendation algorithm. Most of the current research used the model-based collaborative filtering recommendation algorithm. The great success of a hidden factor model in Netflix recommendation system competition makes it one of the important models in the collaborative filtering recommendation algorithm. The basic hidden factor model is the low-rank matrix decomposition model BASEMF [22], which maps the features of users and items into low-dimensional space in the form of vectors. Based on the basic matrix decomposition algorithm, nonnegative matrix factorization (NMF) [23], maximum margin matrix factorization (MMMF) [24], and so on are also derived. Although the basic matrix decomposition model adds an L-2 penalty term to the objective function to prevent overfitting in the process of training, the fitting effect is still not ideal when the training samples are highly sparse. Therefore, researchers propose a probabilistic matrix factorization (PMF) [25] model to model the problem from the perspective of probability, in order to predict the overall sample from the observed sample, so as to alleviate the overfitting problem. Reference [26] further proposed Bayesian PMF (BPMF) on the basis of PMF. The main improvement of BPMF is the introduction of a priori user implicit factor matrix and item implicit factor matrix. Literature [27] proposed a distributed algorithm for largescale datasets. References $[28,29]$ proposed two matrix decomposition frameworks based on general distribution. Good scalability makes PMF and its related models become an important model in the collaborative filtering recommendation algorithm and has achieved good application results.

With the rapid development of Internet technology, the problem of information overload is becoming more and more serious, and the $5 \mathrm{G}$ precision marketing recommendation system came into being [30]. The $5 \mathrm{G}$ precision marketing recommendation system is mainly composed of a user modelling module, recommendation object modelling module, and recommendation algorithm module, in which the recommendation algorithm is the core of the recommendation system. At present, the most widely used recommendation algorithm is the collaborative filtering recommendation algorithm. However, the collaborative filtering recommendation algorithm has unavoidable problems such as data sparsity and cold start. Aimed at the sparsity of the collaborative filtering recommendation algorithm, considering the few common scoring items and the lack of user demand information, this paper studies the film recommendation algorithm based on the collaborative filtering recommendation algorithm. Aimed at the lack of user demand information, an improved method of similarity calculation is proposed. In the similarity calculation of the UBCF recommendation algorithm, demographic information is used to integrate the demographic similarity into the traditional similarity calculation to find the nearest neighbour users more similar to the target users, so as to improve the recommendation quality. The main contributions are summarized as follows: (1) in order to alleviate sparsity and improve dynamic timeliness and recommendation quality, 5G multimedia precision marketing based on the improved multisensor node collaborative filtering recommendation algorithm is proposed in this paper. (2) In the similarity calculation of the UBCF recommendation algorithm, demographic information is used to integrate the demographic similarity into the traditional similarity calculation to find the nearest neighbour users. In this way, the data sparsity is solved. (3) The effectiveness of the above-recommended methods is verified by experimental methods. 


\section{The Design of the 5G Multimedia Precision Marketing System Based on Multisensor Nodes}

2.1. The Structure Design of the 5G Multimedia Precision Marketing System. The structure design of the 5G multimedia precision marketing system is shown in Figure 1. The multimedia precision marketing system mainly includes a sensor layer, network layer, and application layer.

(1) The sensing layer uses multisensor node network technology to collect basic information. The main function is to identify objects and collect information through sensing equipment

(2) The network layer is mainly used to serve the network equipment and platform for aggregation, transmission, and preliminary processing of data collected from the sensor layer. Through the existing three networks (Internet, radio and television network, and communication network), seamless remote transmission of a large amount of data information is perceived by sensors. It is responsible for transmitting the information collected by the sensor safely, analysing and processing the collected information, and then providing the results to the application layer. At the same time, the application of network layer "cloud computing" technology ensures the establishment of practical, applicable, reliable, and efficient information system and intelligent information sharing platform to realize the sharing and optimal management of various information resources

(3) The application layer mainly solves the problems of information processing and man-machine interface, that is, input and output control terminals, such as controllers of mobile phones and smart appliances, mainly providing the information services people need through data processing and solutions. The relevant applications and services of the application layer are directly oriented to users and mainly provide users with personalized service requirements. Users can customize their own personalized services according to the relevant applications of the application layer, such as querying relevant information, monitoring relevant information, and controlling relevant information

2.2. Design of the General Recommendation System Model. The recommendation system is a system used by ecommerce websites to provide users with commodity information and purchase suggestions. It can simulate the salesperson and recommend what products the customer should buy according to the customer's needs. Generally, the recommendation system can be divided into three modules: user modelling, recommendation object modelling, and recommendation algorithm. The core module of the recommendation system is the recommendation algorithm. The general recommendation system model is designed in Figure 2.
(1) User modelling module: establish a user model according to user information. Analyse the user's attribute information, user input information, and user behaviour information (such as historical browsing behaviour and mouse click behaviour), and mine a computable and formal user model from these information and continuously track and update the user model in time. (2) Recommendation object modelling: the description method of recommendation objects will affect the recommendation results. Different recommendation objects have different feature expressions, so the description methods and recommendation results are different. For example, text, which is mainly text symbols, is easier to extract features than $5 \mathrm{G}$ multimedia marketing, so the model is closer to the user interest model. Nowadays, the mainstream recommended object description methods are a content-based method and a classificationbased method. Take the text as an example to briefly summarize these two recommended object modelling methods. When using the content-based method to model the text, first use the feature extraction method to find the keyword of the text, usually use the information increment method to determine the keyword vector, then use the TF-IDF method to select the weight of the keyword, and use Bayesian to recommend. (3) The recommendation algorithm is the core of the recommendation system. The quality of the recommendation algorithm directly determines the recommendation quality of the recommendation system. This paper also focuses on the research of the recommendation algorithm and puts forward effective improvement methods for the problems existing in the current recommendation algorithm

\section{The Design of the Improved Multisensor Node Collaborative Filtering Recommendation Algorithm}

3.1. Collaborative Filtering Matrix and Potential Similarity. With the development of cloud computing, data storage and computing, data sharing, and data application technology, today's era has entered the era of big data. Based on the development of the above technologies, big data technology and big data application have developed rapidly. At present, data information has become a powerful competitiveness of enterprises in market competition and an advantageous asset of enterprises. Therefore, collaborative filtering of effective information from a large amount of data and the use of effective information are the key to precision marketing.

In order to solve the above problems, this section proposes a new prediction mechanism to predict the scores of items that the target users may be interested in through the organic integration of extreme scoring behaviour, nearest neighbour relationship, and recommendation method based on matrix decomposition. Specifically, by introducing extreme rating branches, in order to alleviate the failure of similarity measurement and the instability of the nearest neighbour recommendation in the context of sparse data, the robustness of recommendation performance is further 


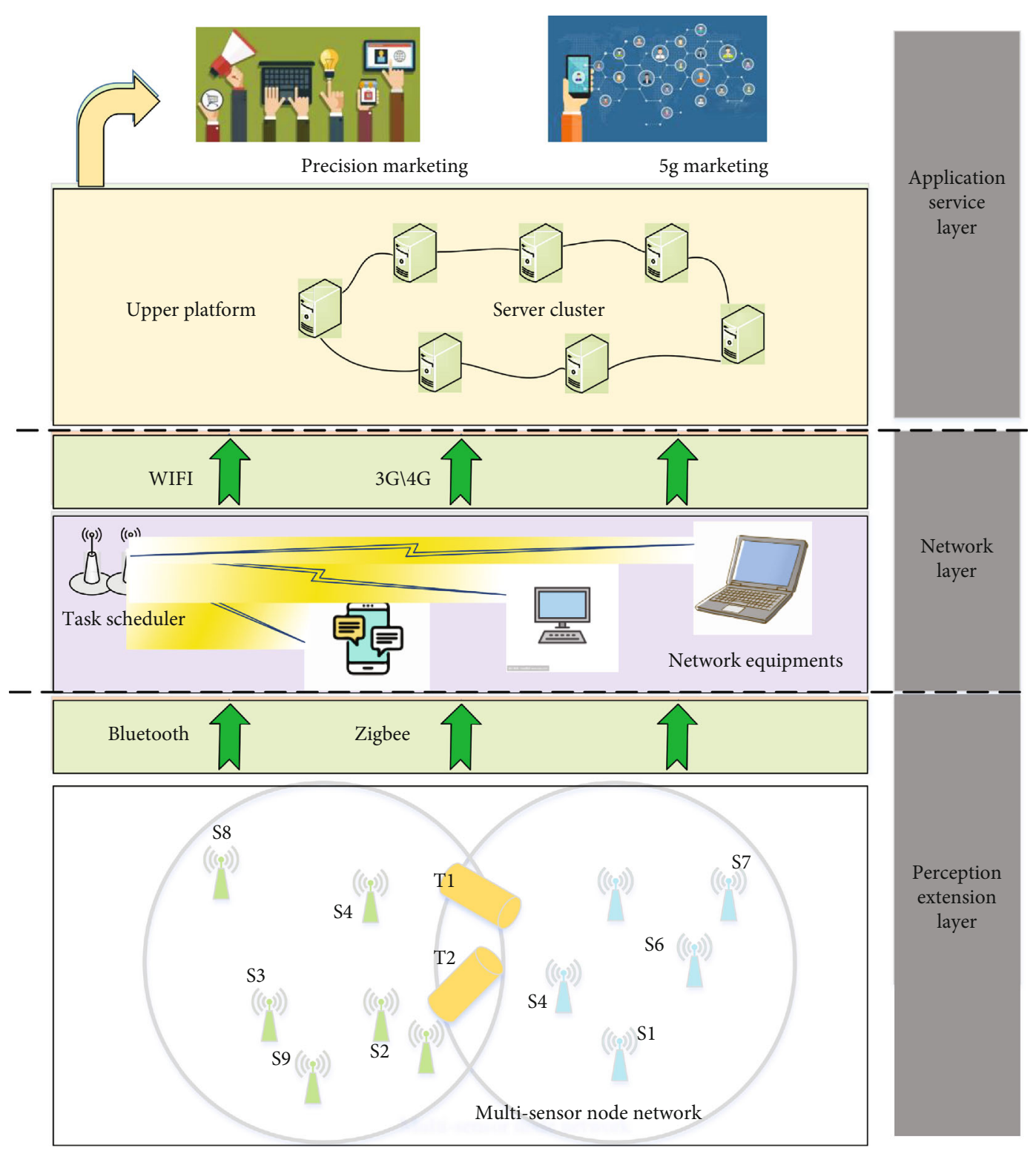

FIGURE 1: The structure of the $5 \mathrm{G}$ multimedia precision marketing system based on multisensor nodes.

improved through the introduction of the matrix decomposition method. It should be noted that probabilistic matrix decomposition is a kind of matrix decomposition method, which is consistent with the optimization objective function of regularized SVD (i.e., singular value decomposition model in the matrix decomposition method). However, considering that the probabilistic matrix decomposition model can more clearly show its prediction mechanism, the matrix decomposition method in this section selects the probability matrix decomposition model. Based on the above considerations, this paper proposes a recommendation method framework considering both extreme scoring behaviour similarity and scoring matrix information fusion. This framework integrates the user's local nearest neighbour relationship into the global scoring optimization process of matrix decomposition. It improves the prediction accuracy and robustness in sparse data situation. The knowledge matrix of collaborative filtering is shown in Figure 3. As shown in Figure 3, first of all, through implicit acquisition, the system "secretly" tracks the user's behavior and records the information that can reflect the user's interest, such as browsing records, query records, mouse clicks, and page dwell time, to record the user's preferences. Secondly, build the knowledge structure between users and preferences. Finally, build the user's scoring system and its matrix to the user's precision marketing in the next step.

The CF algorithm originated from If technology and is the most successful method to realize the recommendation system at this stage. The core idea of CF is to recommend from the perspective of people related to users. Use the scoring matrix and relevant calculation methods to predict the user's score on an item and complete the recommendation. The CF algorithm filters items through the quantitative scoring matrix of user experience and selects items that are difficult to describe (such as movies, music, and pictures) and concepts that are difficult to express (such as tastes and 


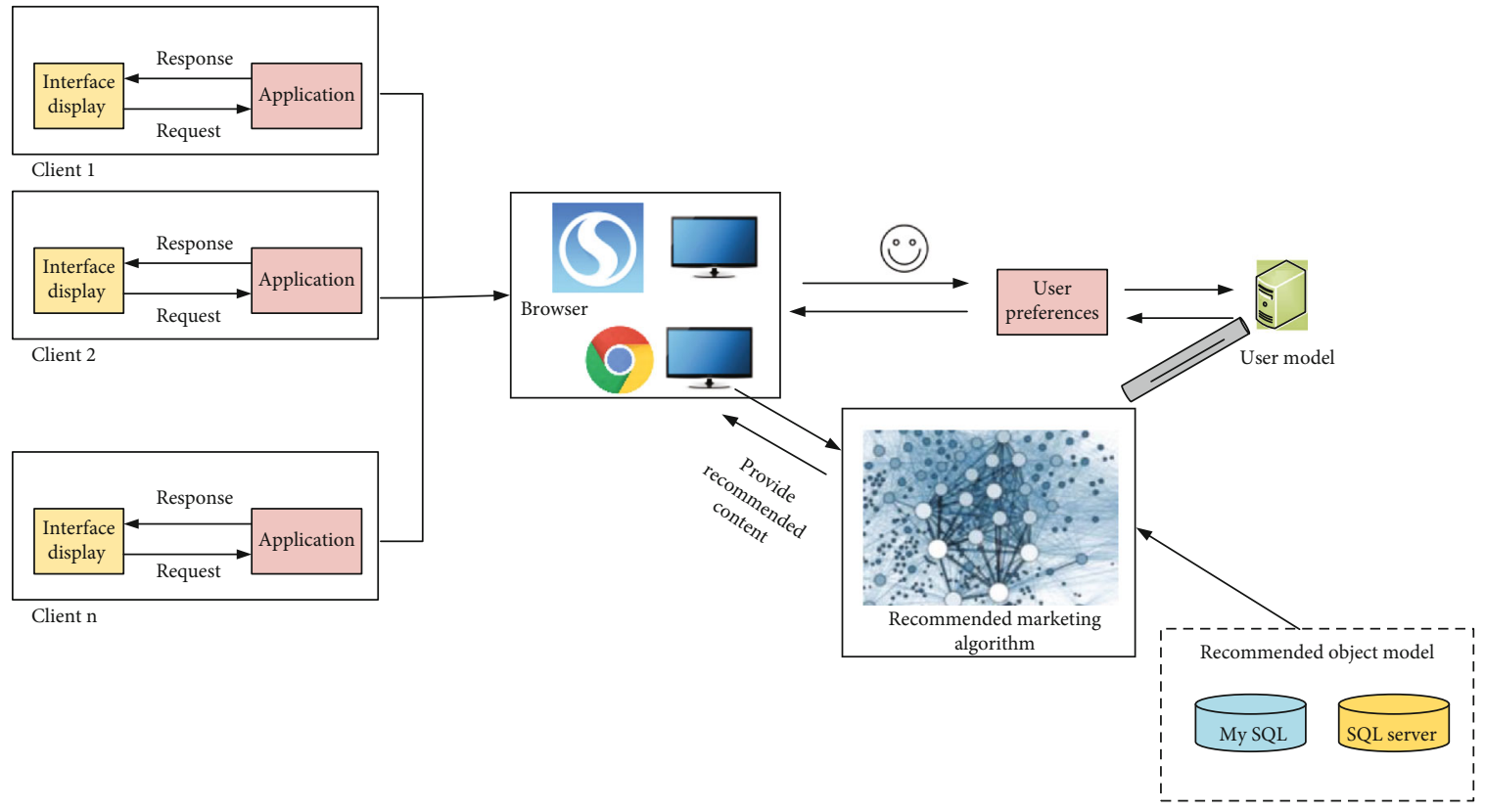

FIgURE 2: Recommended system structure.

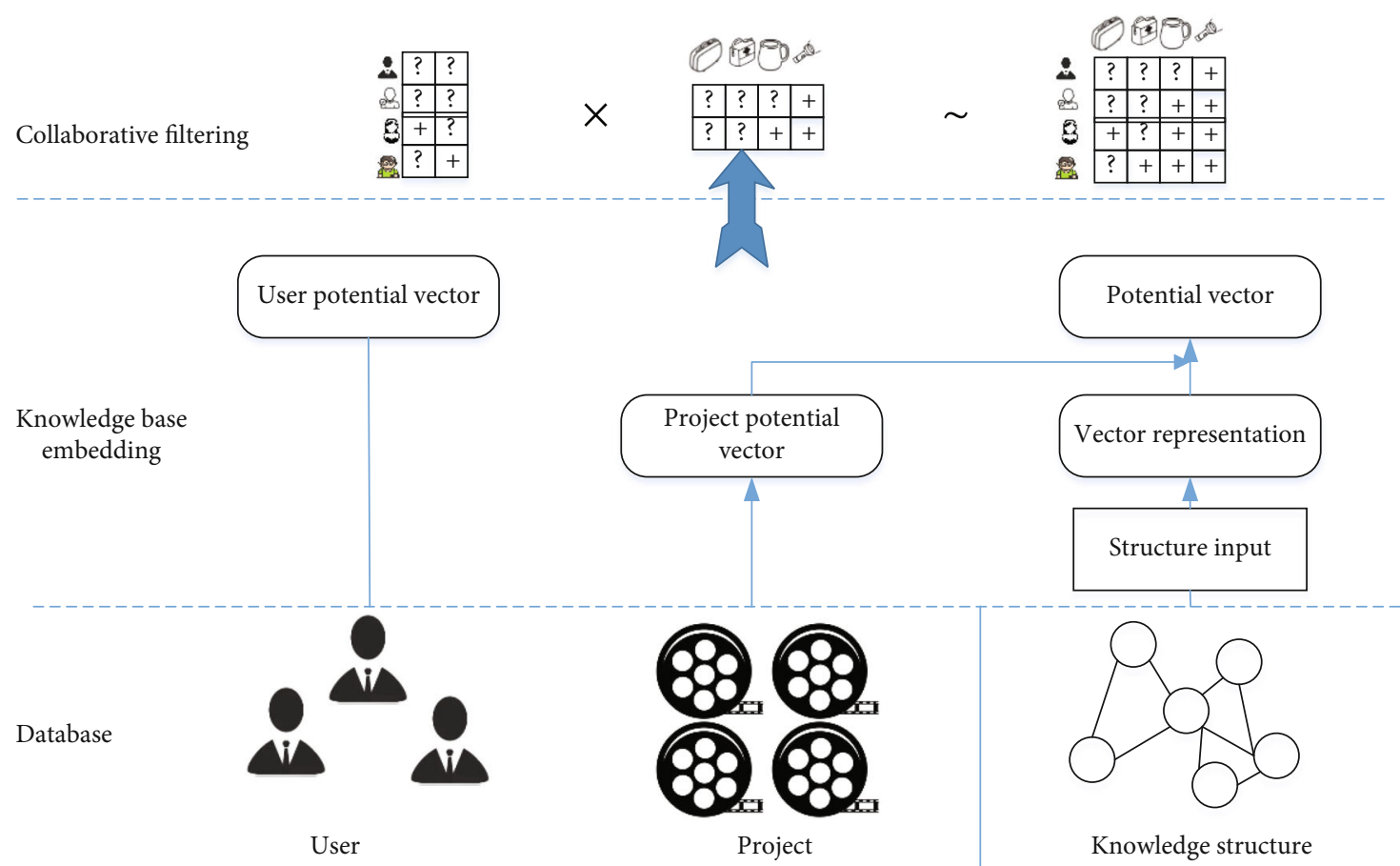

FIgURE 3: Knowledge architecture of the collaborative filtering algorithm.

hobbies). Compared with traditional filtering methods, it can screen out innovative items and truly achieve the purpose of personalized service. For a collaborative filtering recommendation system with $n$ users and $m$ movies, $R_{m \times n}$ indicates its score matrix. $\mathrm{Spa}(R)$ is the score matrix; therefore,

$$
\operatorname{Spa}(R)=\frac{\left|r_{\text {in }}\right| r_{\mathrm{iN}} \in R, r_{i w}=0,1 \leq i \leq n \mid}{n \times m} \times 100 \%,
$$

where the numerator represents the number of " 0 " in the scoring matrix and the denominator represents the number of elements in the scoring matrix.

It is the process of predicting the unknown user score value according to the known user score value. However, in practice, the score matrix $R_{m \times n}$ is an extremely sparse matrix, and there is still a big gap between the predicted value and the user's real preferences. Finding the nearest neighbour is actually the process of establishing the 


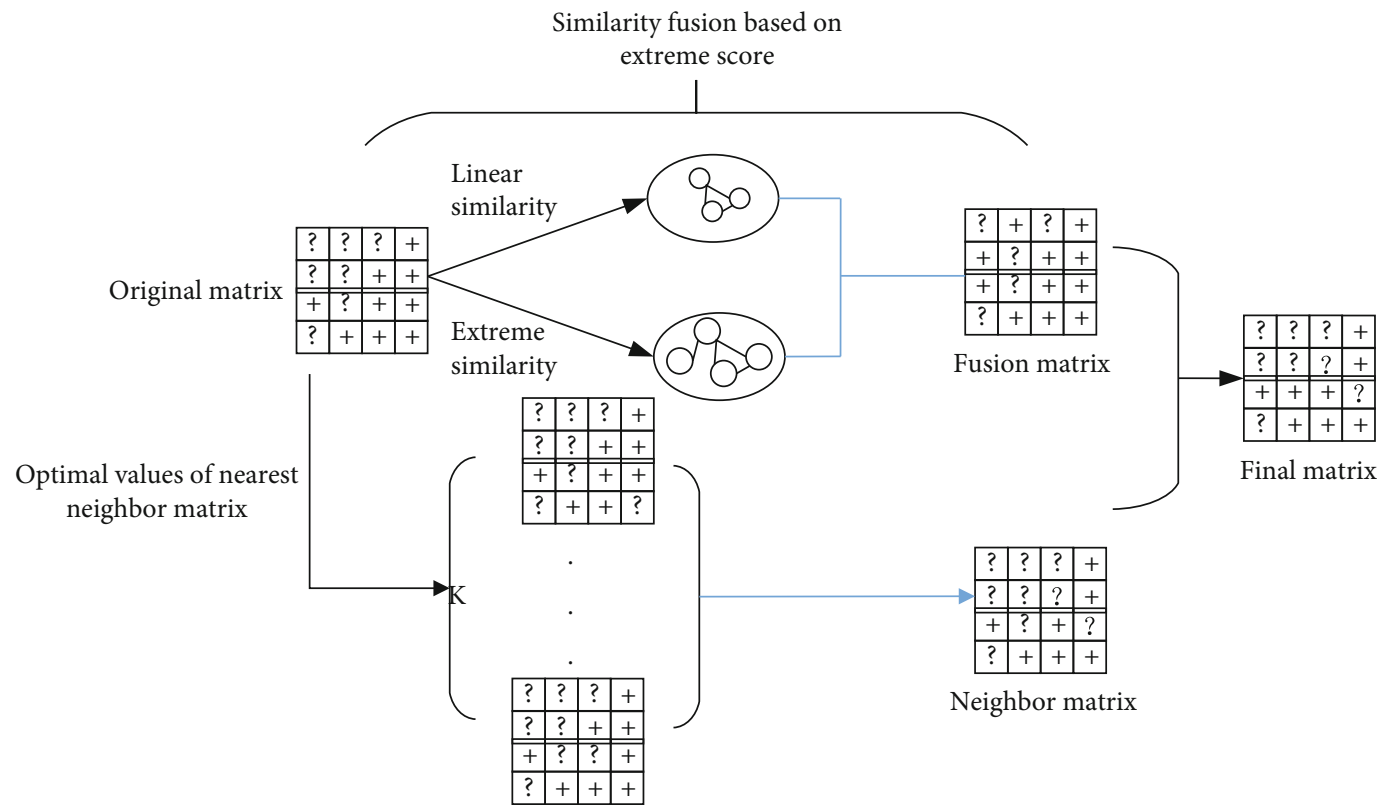

FIgURE 4: Structure of the improved collaborative filtering model.

algorithm model of UBCF, that is, the process of finding users similar to the target user according to the user's scoring behaviour.

Using Euclidean distance to measure the absolute distance between multidimensional vectors is the most common distance measurement method. Distance similarity believes that the smaller the distance between users, the more similar the users are. The distance similarity $\operatorname{sim}(i, j)$ between user $i$ and user $j$ is

$$
\operatorname{sim}(i, j)=d(\vec{i}, \vec{j})=\sqrt{\sum_{k=1}^{m}\left(r_{i k}-r_{j k}\right)^{2}},
$$

where $\vec{i}, \vec{j}$ represents the historical score vector of user $i$ and user $j, m$ is the dimension of the matrix, and $r_{i k}$ and $r_{j k}$ are the score matrix.

The CF algorithm based on the association rule is based on the association rule mining algorithm, which takes the items liked or purchased by the target user as the rule header (LHS) and the rule body (RHS) as the pending recommendation object. The support or confidence of association rules can be used as the similarity between items to find the nearest neighbour, and then, the final recommendation set can be obtained.

If itemset $B$ also appears more in the scene where itemset $A$ appears, it is considered to express an association rule $A$ $\Rightarrow B$. Itemset $A$ is LHS and itemset $B$ is RHS. For all itemsets $I=\left\{i_{1}, i_{2}, \cdots, i_{N}\right\}$ assuming that the set of database transactions is dataset $D$ and the set of items is $t_{m}$, there is $t_{m} \in I$ for $\forall m$. Let $A$ be a set of items with $A \in t_{m}$. An association rule $A \Rightarrow B$, where $A \subseteq I, B \subseteq I$, and $A \cup B=\varnothing$.

Define the proportion of the union set of itemset $A$ and itemset $B$ contained in the transaction with the support degree of $D$ in dataset $D$; there is
TABLE 1: The features of datasets.

\begin{tabular}{lcccc}
\hline Datasets & $\begin{array}{c}\text { User } \\
\text { number }\end{array}$ & $\begin{array}{c}\text { Project } \\
\text { number }\end{array}$ & $\begin{array}{c}\text { Score } \\
\text { number }\end{array}$ & $\begin{array}{c}\text { Density } \\
(\%)\end{array}$ \\
\hline Ml-100k & 943 & 1680 & 100000 & 6.3 \\
ML-latest-small & 1508 & 2071 & 35497 & 1.1 \\
Filmtrust & 671 & 9058 & 100004 & 1.6 \\
CiaoDVD & 17615 & 16121 & 72665 & 0.03 \\
\hline
\end{tabular}

$$
\sup (A \Rightarrow B)=P(A \cup B)=\frac{\operatorname{count}(A \cup B)}{|D|}
$$

Similarly, the confidence of the defining rule $A \Rightarrow B$ in dataset $D$ is the proportion of transactions that contain itemset $A$ and itemset $B$ in $D$; there is

$$
\operatorname{con}(A \Rightarrow B)=P(B \mid A)=\frac{\sup (A \cup B)}{\sup (A)}=\frac{\operatorname{count}(A \cup B)}{\operatorname{count}(A)}
$$

After the confidence is obtained, the confidence is defined as the similarity between items:

$$
\operatorname{sim}(A, B)=\operatorname{con}(A \Rightarrow B)
$$

3.2. The Improved Collaborative Filtering Recommendation Algorithm. The improved collaborative filtering model (ICF model) includes three parts: (1) extreme scoring similarity is introduced to overcome the shortcomings of traditional similarity measurement. (2) By combining the proposed extreme scoring behaviour similarity with linear similarity, the similarity based on extreme scoring behaviour is established, and then, the user relevance is described more comprehensively. (3) The nearest neighbour matrix is generated by similarity based on extreme scoring behaviour, 


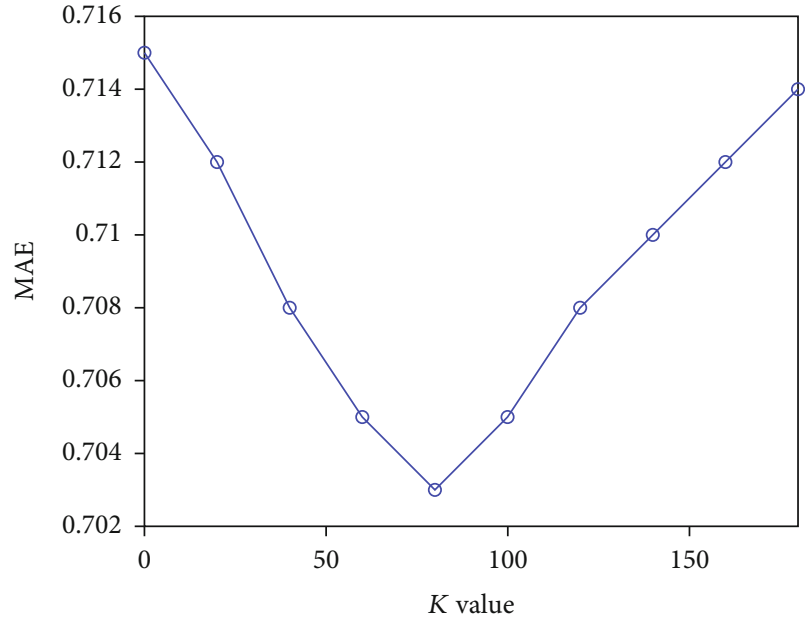

(a) MAE

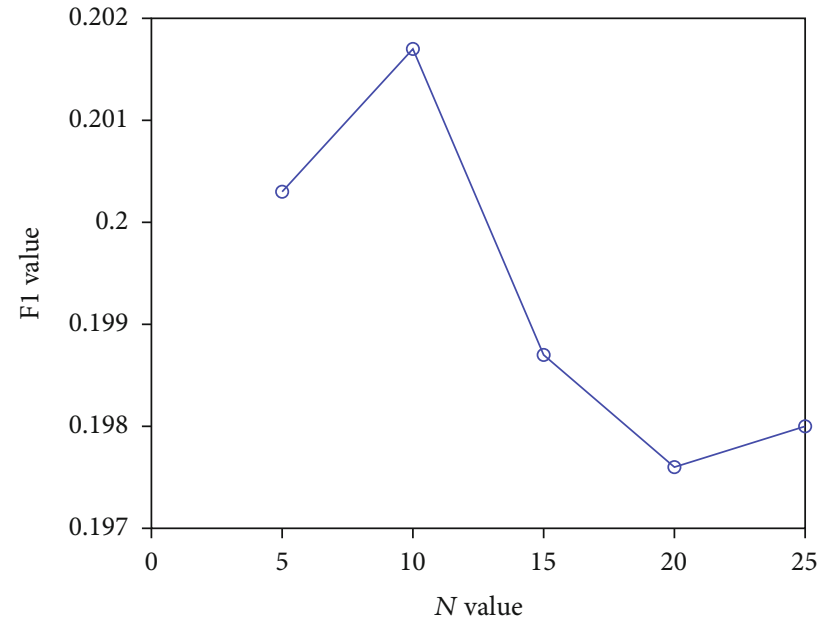

(b) RMSE

FIGURE 5: The structure of the improved sequential pattern mining algorithm.

which is fused into the original scoring matrix to improve the recommendation performance. Figure 4 is the flowchart of the ICF model. The flowchart of the algorithm is as follows: the mixed matrix of the score matrix is obtained by using the similarity theory and the limit theory. By selecting the adjacent $K$ score matrices to solve the average, the similarity matrix is obtained. The final scoring matrix can be obtained by fusing the mixing matrix with the similarity matrix.

The probability matrix decomposition model uses the Bayesian inference method to convert the posterior probability into the product of a priori and likelihood function. Its calculation scale is linear with the number of observations. More importantly, it performs well on large-scale, sparse, and unbalanced datasets. The probability matrix decomposition model assumes that the conditional probability distribution (likelihood function) obeyed by the known score $R$ is

$$
P\left(R \mid A, B, \sigma^{2}\right)=\prod_{i=1}^{m} \prod_{j=1}^{n}\left[N\left(r_{i j} \mid A_{i}^{T} B_{j}, \sigma^{2}\right)\right]^{I_{i j}}
$$

By assuming that the potential eigenvectors of users and projects obey the mean value of zero, the variance is $\sigma_{A}^{2}$ and $\sigma_{B}^{2}$ Gaussian priori:

$$
\left\{\begin{array}{l}
p\left(A \mid \sigma_{A}^{2}\right)=\prod_{i=1}^{n}\left(N\left(A_{i} \mid 0, \sigma_{A}^{2} I\right)\right), \\
p\left(B \mid \sigma_{A}^{2}\right)=\prod_{i=1}^{n}\left(N\left(B_{i} \mid 0, \sigma_{B}^{2} I\right)\right) .
\end{array}\right.
$$

The maximum log a posteriori probability is equivalent to the minimum sum of squares of error function with regularization:

$$
E=\frac{1}{2} \sum_{i=1}^{m} \sum_{j=1}^{n} I_{i j}\left(r_{i j}-A_{i}^{T} B_{j}\right)+\frac{\lambda_{A}}{2} \sum_{i=1}^{m}\left\|A_{i}\right\|^{2}+\frac{\lambda_{B}}{2} \sum_{j=1}^{n}\left\|B_{i}\right\|^{2},
$$

where $\lambda_{A}$ and $\lambda_{B}$ are the parameters of the regularization weight.

\section{Simulation Results and Performance Analysis}

4.1. Data Sources and Simulation Setting. With the in-depth study of the recommendation system, major research teams have released a series of recommendation system research and test datasets, for example, the movie lens dataset of the movie recommendation system, the Netflix dataset of the movie rental website Netflix, the jester joke dataset of the joke system, and the user browsing data Usenet newsgroups of the newsgroup. In order to evaluate the performance of the proposed method, we selected four commonly used datasets: Ml-100k, ML-latest-small, Filmtrust, and CiaoDVD. Among them, Ml-100k and ML-latest-small are two representative datasets in the movie lens dataset, which were collected by the GroupLens research group of the University of Minnesota; the scoring range is from 1 to 5. Filmtrust is a dataset captured from the Filmtrust website. Its scoring range is from 0.5 to 4 with an interval of 0.5 . CiaoDVD is a DVD category dataset. The statistical data of scoring ranging from 1 to 5.4 datasets are shown in Table 1, where the density represents the available scoring proportion in the scoring matrix. We usually think that the first three datasets are sparse. The fourth dataset is very sparse.

Since the main purpose of the model is to alleviate the sparsity problem and improve the robustness of the model recommendation performance. In order to verify the effectiveness of the methods, this section randomly deletes $20 \%$, $40 \%, 60 \%$, and $80 \%$ of the scores on the dataset (Ml-100k, ML-latest-small, Filmtrust, and CiaoDVD) and constructs a series of experiments on the sparse dataset. 


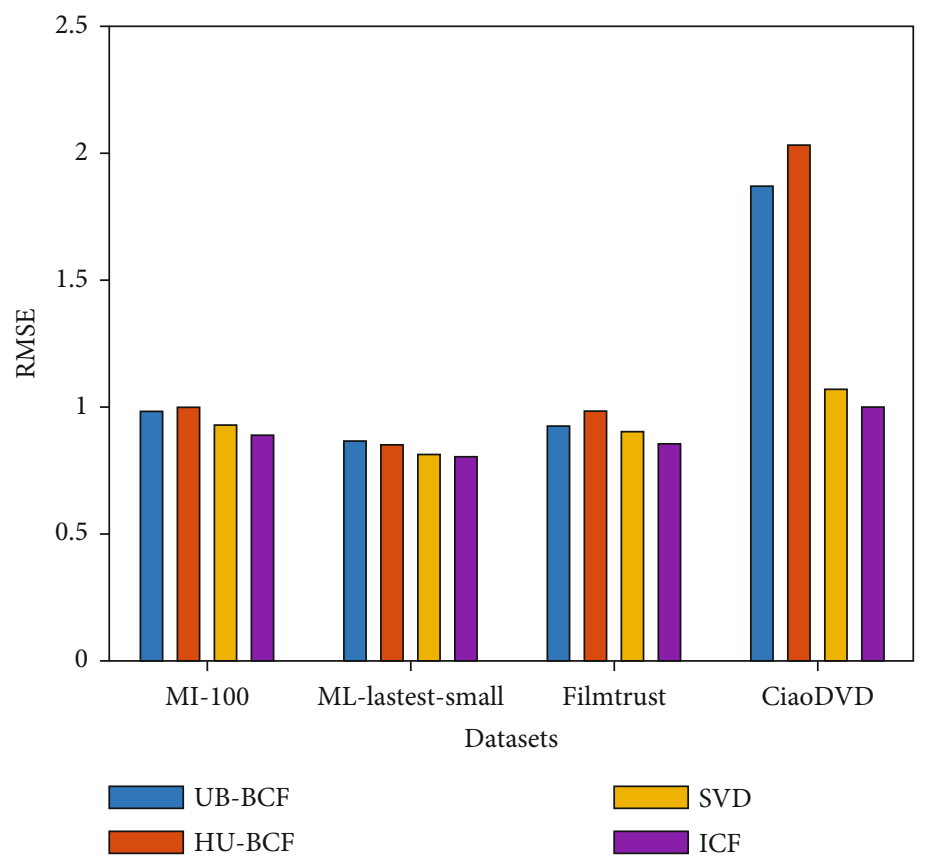

(a) MAE

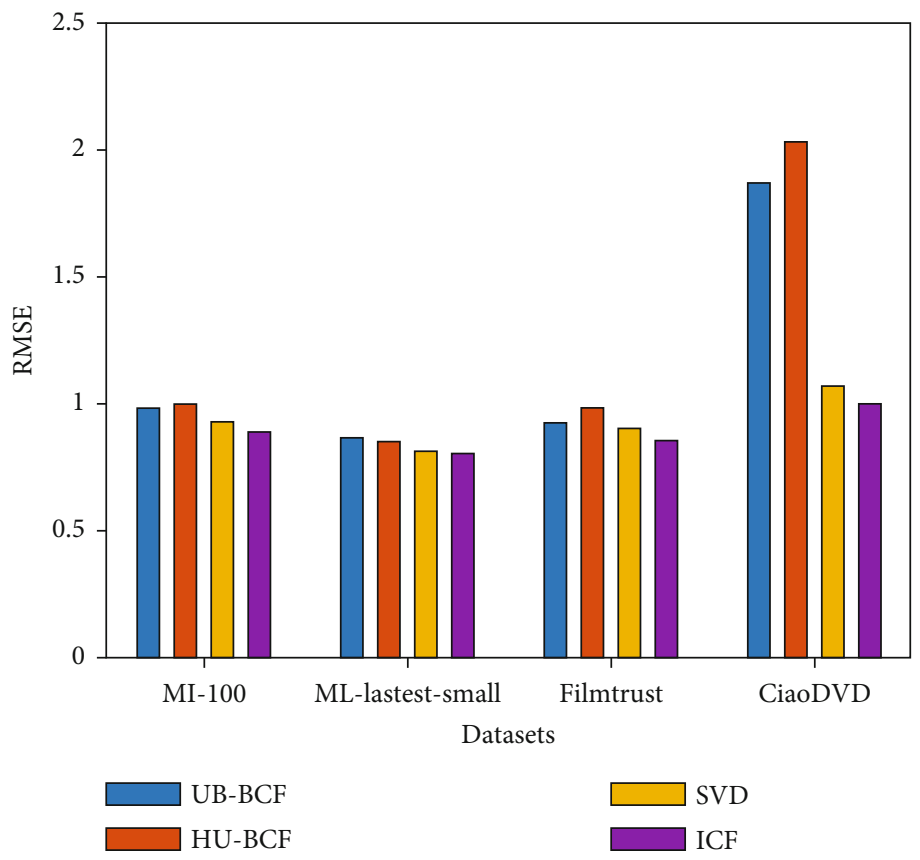

(b) RMSE

FiguRE 6: The structure of the improved sequential pattern mining algorithm.

In order to verify the performance of the proposed method, three groups of experiments are designed: (1) the proposed fusion model is compared with six common collaborative filtering methods. (2) For different sparse datasets, the method in this paper is compared with four common matrix decomposition methods. (3) The fusion model (ICF) in this paper is compared with some other representative similarity measurement fusion models. We use VS2010 to implement the CF and ICF algorithm on a machine with $1024 \mathrm{M}$ memory,
$733 \mathrm{MHz} \mathrm{CPU}$, and Windows 10 operating system. The ICF algorithm is compared with the CF algorithm.

4.2. Optimal Parameter Selection. In order to verify the influence of the number of the nearest neighbours, the number of recommended movies $N$ in the fixed recommendation list is 10 and the user has the least number of jointly rated movies $\varphi$. The number of the nearest neighbours $K$ value is increased from 5 to 160 in a multiple growth mode, and 


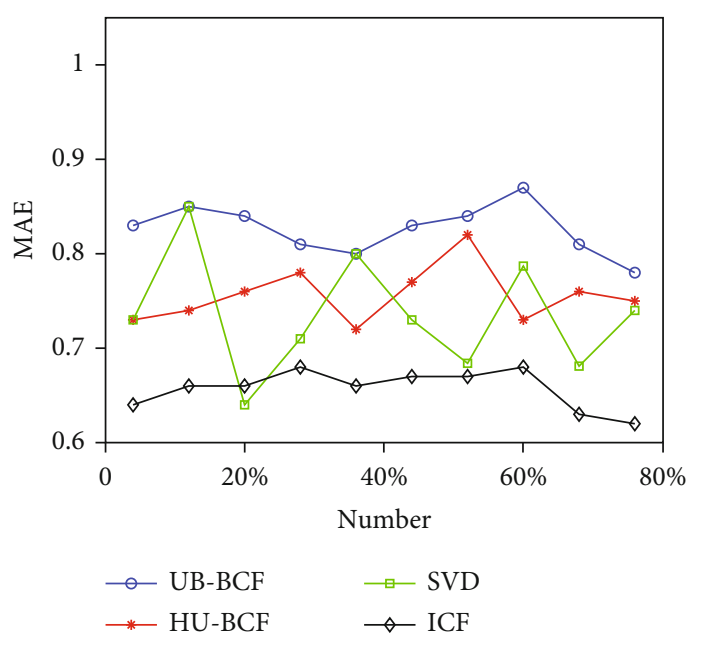

(a) MAE

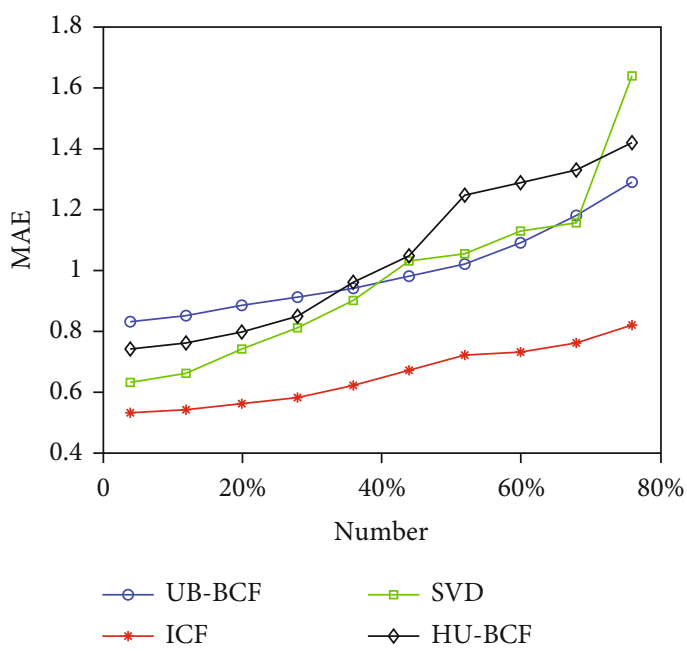

(c) MAE

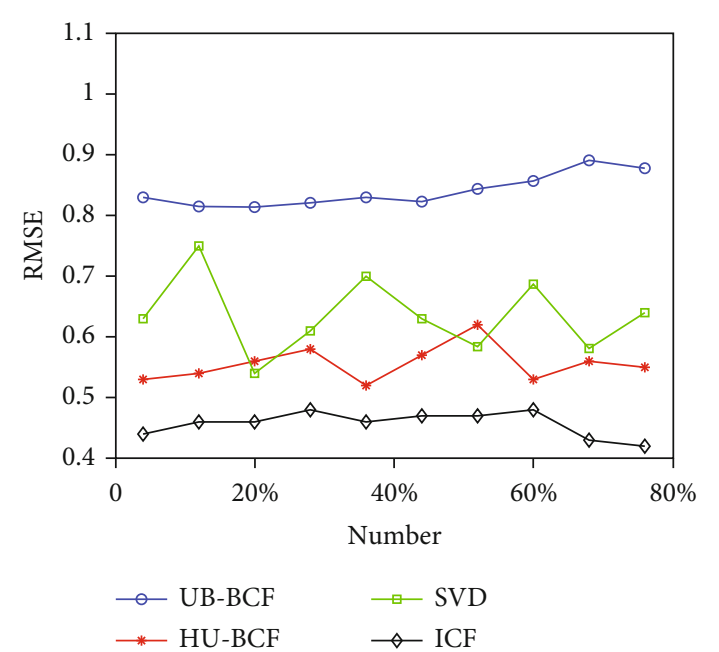

(b) RMSE

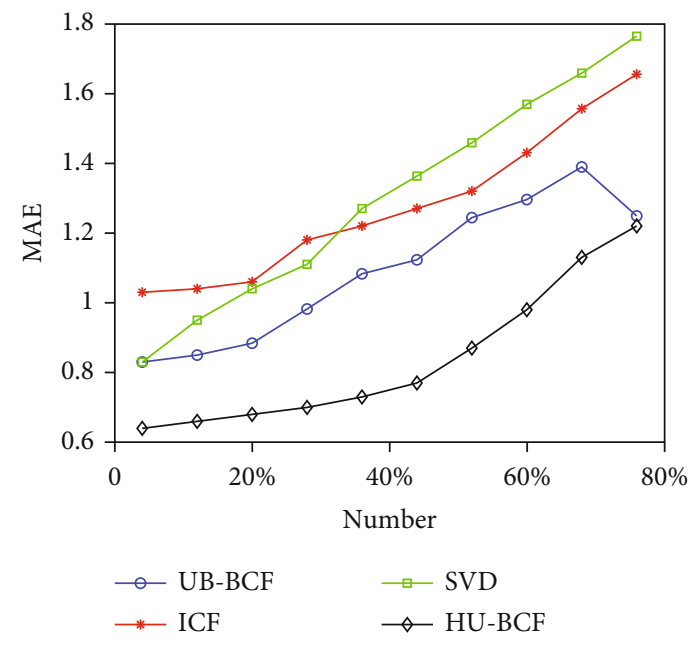

(d) RMSE

FIGURE 7: The simulation results of the proposed method.

the MAE- $K$ curve is drawn to reflect the recommendation effect of the ICF algorithm under different $K$ values. Changing the nearest neighbour number $k$ actually changes the prediction score of the target user. The experimental results show that when the number of the nearest neighbour $K$ increases from 5 to 80 , the MAE value of the ICF algorithm gradually decreases and the recommendation accuracy gradually increases; when the number of the nearest neighbours $K$ increases from 80 to 160 , the MAE value of the ICF algorithm increases and the recommendation accuracy decreases. It can be seen from Figure 5(a) that when the nearest neighbour $K$ is 80 , the MAE value of the ICF algorithm is the smallest and the recommendation effect is the best. Therefore, the optimal $K$ value of this dataset is 80 .

Furthermore, in order to verify the relationship between the number of recommended movies in the recommendation list and the recommendation accuracy of the ICF algorithm, fix the number of the nearest neighbours $K$ as 80 , increase the number of recommended movies $n$ in the recommendation list from 5 to 25 in steps of 5 , and draw the F1-N curve accordingly. Observe the experimental results of the change of the number of recommended movies $n$ in the recommendation list. When the number of recommended movies $n$ increases from 1 to 10, the change trend of F1 increases, indicating that the recommendation accuracy of the ICF algorithm is improved; when the number of recommended movies $n$ increases from 10 to 25 , the change trend of F1 decreases, indicating that the recommendation accuracy of the ICF algorithm decreases gradually. It can be seen from Figure 5(b) that when the number of recommended movies $n$ is 10 , the F1 value of the ICF algorithm is the largest and the recommendation effect is the best. Therefore, the optimal $n$ value of this dataset is 10 .

4.3. Comparative Simulation of Common Collaborative Filtering Methods. The purpose of this experiment is to verify the performance of the proposed model in terms of prediction accuracy. Therefore, this section compares and analyses the proposed ICF recommendation algorithm with three commonly used collaborative filtering recommendation algorithms, such as UB_BCF [30], HU-BCF [31], and SVD [32]. As shown in Figure 6, the proposed ICF model 
is obviously superior to other methods, especially in the sparse CiaoDVD dataset.

On the three datasets of Ml-100k, ML-latest-small, and Filmtrust, the MAE obtained by the ICF model is optimal and is reduced by $0.52 \%, 0.6 \%$, and $0.69 \%$, respectively, compared with the suboptimal method. In Figure 6(b), the RMSE of the ICF model is reduced by $0.11 \%, 0.83 \%$, and $1.54 \%$, respectively, compared with that of the suboptimal method. From the perspective of robustness, the ICF algorithm proposed in this paper shows relatively stable recommendation performance. Other collaborative filtering methods show unstable prediction accuracy. On the CiaoDVD dataset, the recommendation method based on the matrix decomposition model has a better recommendation effect than the user-based nearest neighbour method. The reason is that when the dataset is very sparse, the similarity is very difficult to measure. Overall, the experimental results in this section show that the organic integration of the extreme rating branch, nearest neighbour relationship, and SVD can effectively alleviate the problem of sparsity and improve the robustness of the recommended method in prediction accuracy.

4.4. Comparative Simulation of Different Sparse Levels. The purpose of this experiment is to verify that the ICF model based on the fusion of the local nearest neighbour information and global score information has robustness 5zadvantages in recommendation prediction accuracy under different sparsity situations. Figure 7 shows the MAE and RMSE results at different sparsity levels, in which different sparsity levels are obtained by randomly deleting scores. The results show that, on the datasets, MAE and RMSE increase with the increase in sparsity, which is consistent with our expectation; that is, with the increase in sparsity, the recommendation error will increase. However, the results also show that the MAE and RMSE values of the fusion model proposed in this paper are better than those of other comparison methods at different sparsity levels.

For the Ml-100k dataset, after removing the scores of $20 \%, 40 \%, 60 \%$, and $80 \%$, the MAE of the method in this paper is reduced by $0.68 \%, 1.12 \%, 2.85 \%$, and $1.19 \%$, respectively, compared with that of the suboptimal method. RMSE shows the same change trend. Figure 7 shows that for the datasets Filmtrust and ML-latest-small, the ICF model has slight advantages over other methods in MAE and RMSE when randomly deleting $20 \%, 40 \%$, and $60 \%$ of the scores. However, when $80 \%$ of the scores are deleted, the advantages of the method proposed in this paper further increase on these two datasets. Overall, the ICF model proposed in this chapter has better prediction performance than other methods in different sparse situations. Further, the comparative experiments in this section verify the robustness of the ICF model.

\section{Conclusion}

With the development of Internet, communication, and Internet of things technologies, Internet companies are developing rapidly. The rich content applications and customer-based personalized marketing of Internet companies have brought great challenges to telecom operators. At the same time, the promotion of number carrying network transfer business and the commercialization of $5 \mathrm{G}$ technology have further weakened the advantages of traditional business operation of operators. The lack of exploration of customers' personalized needs, no targeted marketing activities, and the traditional extensive operation do not effectively combine operators' products with users' needs, and the marketing success rate is low, which leads to the crisis that operators may become "pipelines," so the implementation of precision marketing to meet users' personalized needs is imminent. Collaborative filtering is one of the most commonly used methods in recommendation systems. However, accurate prediction of unknown scores is still a challenging problem in the context of sparse data. Based on the user-based nearest neighbour recommendation method and probability matrix decomposition method, combined with similarity measurement based on extreme scoring behaviour, a new collaborative filtering recommendation method is constructed. This method comprehensively considers the local nearest neighbour relationship and global scoring information and establishes a probability matrix decomposition model integrating the nearest neighbour information, which effectively improves the prediction accuracy and robustness.

\section{Data Availability}

The data used to support the findings of this study are available from the corresponding author upon request.

\section{Conflicts of Interest}

The authors declare that they have no known competing financial interests or personal relationships that could have appeared to influence the work reported in this paper.

\section{References}

[1] C. Yin, L. Shi, R. Sun, and J. Wang, "Improved collaborative filtering recommendation algorithm based on differential privacy protection," The Journal of Supercomputing, vol. 76, no. 7, pp. 5161-5174, 2020.

[2] S. Li, X. Song, H. Lu, L. Zeng, M. Shi, and F. Liu, "Friend recommendation for cross marketing in online brand community based on intelligent attention allocation link prediction algorithm," Expert Systems with Applications, vol. 139, article 112839, 2020.

[3] L. Xiaojun, "An improved clustering-based collaborative filtering recommendation algorithm," Cluster Computing, vol. 20, no. 2, pp. 1281-1288, 2017.

[4] M. Jiang, Z. Zhang, J. Jiang, Q. Wang, and Z. Pei, “A collaborative filtering recommendation algorithm based on information theory and bi-clustering," Neural Computing and Applications, vol. 31, no. 12, pp. 8279-8287, 2019.

[5] P. Zhang, Z. Zhang, T. Tian, and Y. Wang, "Collaborative filtering recommendation algorithm integrating time windows and rating predictions," Applied Intelligence, vol. 49, no. 8, pp. 3146-3157, 2019. 
[6] Y. Wang, Z. Song, and C. Xiao, "Collaborative filtering recommendation algorithm based on improved clustering and matrix factorization," Journal of Computer Applications, vol. 38, no. 4, pp. 1001-1006, 2018.

[7] C. Li and K. He, "CBMR: an optimized MapReduce for itembased collaborative filtering recommendation algorithm with empirical analysis," Concurrency and Computation: Practice and Experience, vol. 29, no. 10, article e4092, 2017.

[8] J. Deng, J. Guo, and Y. Wang, "A novel K-medoids clustering recommendation algorithm based on probability distribution for collaborative filtering," Knowledge-Based Systems, vol. 175, pp. 96-106, 2019.

[9] M. Ghazanfari, H. Mohammadi, M. S. Pishvaee, and E. Teimoury, "Fresh-product trade management under government-backed incentives: a case study of fresh flower market," IEEE Transactions on Engineering Management, vol. 66, no. 4, pp. 774-787, 2019.

[10] B. S. Neysiani, N. Soltani, R. Mofidi, and M. H. NadimiShahraki, "Improve performance of association rule-based collaborative filtering recommendation systems using genetic algorithm," International Journal of Information Technology and Computer Science, vol. 11, no. 2, pp. 48-55, 2019.

[11] J. Xu, A. Liu, N. Xiong, T. Wang, and Z. Zuo, "Integrated collaborative filtering recommendation in social cyber-physical systems," International Journal of Distributed Sensor Networks, vol. 13, no. 12, Article ID 1550147717749745, 2017.

[12] M. F. Aljunid and M. Doddaghatta Huchaiah, "Multi-model deep learning approach for collaborative filtering recommendation system," CAAI Transactions on Intelligence Technology, vol. 5, no. 4, pp. 268-275, 2020.

[13] Y. Yun, D. Hooshyar, J. Jo, and H. Lim, "Developing a hybrid collaborative filtering recommendation system with opinion mining on purchase review," Journal of Information Science, vol. 44, no. 3, pp. 331-344, 2018.

[14] C. Zhang, M. Yang, J. Lv, and W. Yang, “An improved hybrid collaborative filtering algorithm based on tags and time factor," Big Data Mining and Analytics, vol. 1, no. 2, pp. 128-136, 2018.

[15] M. Jalili, S. Ahmadian, M. Izadi, P. Moradi, and M. Salehi, "Evaluating collaborative filtering recommender algorithms: a survey," IEEE access, vol. 6, pp. 74003-74024, 2018.

[16] Z. Cui, X. Xu, F. Xue et al., "Personalized recommendation system based on collaborative filtering for IoT scenarios," IEEE Transactions on Services Computing, vol. 13, no. 4, pp. 685695, 2020.

[17] Y. Liu, "Data mining of university library management based on improved collaborative filtering association rules algorithm," Wireless Personal Communications, vol. 102, no. 4, pp. 3781-3790, 2018.

[18] M. He, M. Chang, and X. Wu, "A collaborative filtering recommendation method based on differential privacy," Journal of Computer Research and Development, vol. 54, no. 7, p. 1439, 2017.

[19] P. Kumar, V. Kumar, and R. S. Thakur, "A new approach for rating prediction system using collaborative filtering," Iran Journal of Computer Science, vol. 2, no. 2, pp. 81-87, 2019.

[20] M. Nilashi, O. Ibrahim, and K. Bagherifard, "A recommender system based on collaborative filtering using ontology and dimensionality reduction techniques," Expert Systems with Applications, vol. 92, pp. 507-520, 2018.

[21] R. Chen, Q. Hua, Y. S. Chang, B. Wang, L. Zhang, and X. Kong, "A survey of collaborative filtering-based recommender sys- tems: from traditional methods to hybrid methods based on social networks," IEEE Access, vol. 6, pp. 64301-64320, 2018.

[22] N. Nassar, A. Jafar, and Y. Rahhal, "A novel deep multi-criteria collaborative filtering model for recommendation system," Knowledge-Based Systems, vol. 187, article 104811, 2020.

[23] H. Li, X. Diao, J. Cao, and Q. Zheng, "Collaborative filtering recommendation based on all-weighted matrix factorization and fast optimization," Ieee Access, vol. 6, pp. 25248-25260, 2018.

[24] M. K. Najafabadi, A. Mohamed, and C. W. Onn, "An impact of time and item influencer in collaborative filtering recommendations using graph-based model," Information Processing \& Management, vol. 56, no. 3, pp. 526-540, 2019.

[25] T. Cunha, C. Soares, and A. C. de Carvalho, "Metalearning and recommender systems: a literature review and empirical study on the algorithm selection problem for collaborative filtering," Information Sciences, vol. 423, pp. 128-144, 2018.

[26] R. Logesh, V. Subramaniyaswamy, D. Malathi, N. Sivaramakrishnan, and V. Vijayakumar, "Enhancing recommendation stability of collaborative filtering recommender system through bio-inspired clustering ensemble method," Neural Computing and Applications, vol. 32, no. 7, pp. 21412164, 2020.

[27] X. Yu, F. Jiang, J. du, and D. Gong, “A cross-domain collaborative filtering algorithm with expanding user and item features via the latent factor space of auxiliary domains," Pattern Recognition, vol. 94, pp. 96-109, 2019.

[28] S. Mandal and A. Maiti, "Deep collaborative filtering with social promoter score-based user-item interaction: a new perspective in recommendation," Applied Intelligence, vol. 51, no. 11, pp. 7855-7880, 2021.

[29] Z. Chen, Y. Wang, S. Zhang, H. Zhong, and L. Chen, "Differentially private user-based collaborative filtering recommendation based on K-means clustering," Expert Systems with Applications, vol. 168, article 114366, 2021.

[30] D. Murnane, M. White, and A. G. Williams, "Exploring fine-tuning of the next-to-minimal composite Higgs model," Journal of High Energy Physics, vol. 2019, no. 4, pp. 1-27, 2019.

[31] F. Zhang, V. E. Lee, R. Jin et al., "Privacy-aware smart city: a case study in collaborative filtering recommender systems," Journal of Parallel and Distributed Computing, vol. 127, pp. 145-159, 2019.

[32] S. Yang, M. Korayem, K. AlJadda, T. Grainger, and S. Natarajan, "Combining content-based and collaborative filtering for job recommendation system: a cost-sensitive statistical relational learning approach," Knowledge-Based Systems, vol. 136, pp. 37-45, 2017. 